\section{Utilização de medicamentos por aposentados brasileiros. 1 - Metodologia e resultados de cobertura de inquérito multicêntrico}

\author{
Use of medications by elderly Brazilian retirees. \\ 1 - Methodology and response rates \\ of a multicenter survey
}

\author{
1 Faculdade de Farmácia, \\ Universidade Federal \\ de Minas Gerais, \\ Belo Horizonte, Brasil. \\ 2 Escola Nacional de Saúde \\ Pública Sergio Arouca, \\ Fundação Oswaldo Cruz, \\ Rio de Janeiro, Brasil. \\ Correspondência \\ C. H. Klein \\ Departamento de \\ Epidemiologia e Métodos \\ Quantitativos em Saúde, \\ Escola Nacional de Saúde \\ Pública Sergio Arouca, \\ Fundação Oswaldo Cruz. \\ Rua Leopoldo Bulhões 1480, \\ Rio de Janeiro, $R J$ \\ 21041-210, Brasil. \\ chklein@ensp.fiocruz.br
}

\section{Abstract}

This paper reports methodological aspects and response rates of a survey with the main objective of describing the drug utilization profile of retirees of the Institute of National Social Security of Brazil, 60 years of age or older, in three simple random samples: Brazil (countrywide), Belo Horizonte municipality, and Rio de Janeiro municipality. This cross-sectional study employed two approaches: mail (self-report questionnaires) and home interviews (questionnaires completed by interviewers). This strategy allowed evaluation of response agreement between the mail and home interview approaches. In the national sample of 3,000 individuals, 1,025 only responded to the self-report questionnaire. Despite this the low response rate, there was reasonable resemblance between respondents and non-respondents, suggesting that this sample is representative of the target population. In Belo Horizonte and Rio de Janeiro the initial samples of 800 individuals were replicated for both approaches, mail and home interview. The response rates to the mail surveys were 46.8\% and 34.4\% in Belo Horizonte and Rio de Janeiro, respectively. The response rates to the home interviews were $80.3 \%$ and $70.7 \%$ in Belo Horizonte and Rio de Janeiro, respectively, after unavoidable attrition.

Interviews; Aging Health; Drugs Utilization
Francisco de Assis Acurcio ${ }^{1}$

Suely Rozenfeld 2

Andréia Queiroz Ribeiro 1

Carlos Henrique Klein 2

Cristiano Soares de Moura 1

Célia Regina de Andrade 2

\section{Introdução}

No recenseamento do ano de 2000, o Instituto Brasileiro de Geografia e Estatística (População: Censo Demográfico 2000 - Resultado do Universo. http://www.ibge.gov.br, acessado em 20/Out/2004) contou 14.536.029 idosos residentes no Brasil, ou seja, com 60 anos ou mais de idade. A cada ano, mais de 650 mil idosos são incorporados à população brasileira 1 . Em 2002, o país contava com mais de 20 milhões de aposentados, pensionistas e beneficiários do Instituto Nacional de Seguridade Social (INSS), Ministério da Previdência Social (MPAS) 2, dentre os quais, aproximadamente metade era idosa. A demanda deste grupo por recursos de saúde é intensa, no que se refere tanto à utilização de serviços médicos, quanto ao uso de medicamentos.

A população idosa apresenta peculiaridades em relação ao uso de medicamentos. Alterações fisiológicas decorrentes do processo de envelhecimento interferem na farmacocinética e na farmacodinâmica dos medicamentos e fazem com que eles atuem de forma diferente do que ocorre com adultos jovens, podendo ocasionar ausência de efeitos farmacológicos esperados, bem como aumento da freqüência de reações adversas. As doenças próprias da terceira idade produzem um padrão de prescrição e de uso dos medicamentos diferente daquele encontrado em outras faixas etárias 3 . Além disso, o aces- 
so a medicamentos se constitui em um dos grandes problemas vivenciados pelos idosos.

Inquéritos populacionais direcionados para informações sobre a utilização de medicamentos por idosos são importantes para a identificação de problemas existentes entre os grupos mais vulneráveis, bem como de fatores associados a estes problemas, de modo a fornecer subsídios para o seu adequado enfrentamento. No Brasil, poucos estudos epidemiológicos sobre consumo de medicamentos têm sido feitos, e, ainda assim, todos limitados a populações restritas a um único município 4,5,6,7,8,9. Também não há registro de inquéritos de autopreenchimento, encaminhados por via postal, sobre este tema. A replicação de amostras para abordagens de entrevistas pelas vias postal e domiciliar poderá contribuir para avaliar o grau de concordância das respostas obtidas.

O presente estudo é conseqüência de uma iniciativa da Confederação Brasileira de Aposentados e Pensionistas (COBAP), a qual manifestou ao Ministério da Saúde interesse em colaborar com uma pesquisa sobre o perfil de consumo de medicamentos, a fim de que os resultados pudessem servir de subsídios para o aperfeiçoamento da política nacional de medicamentos.

O objetivo principal do estudo era descrever o perfil de utilização de medicamentos por aposentados e pensionistas do INSS, de 60 anos ou mais, em três amostras: do Brasil e dos municípios de Belo Horizonte (Minas Gerais) e do Rio de Janeiro. Era desejado também descrever os medicamentos utilizados, caracterizar seu uso, determinar os problemas mais importantes para sua obtenção e investigar associações entre os padrões de sua utilização e variáveis sócio-demográficas relativas aos usuários. Por fim, desejava-se ainda comparar coberturas e resultados obtidos por meio de duas abordagens, postal e domiciliar, para coleta de informações, utilizadas nos municípios de Belo Horizonte e do Rio de Janeiro.

Este artigo irá descrever somente a metodologia geral utilizada no estudo e os resultados da sua aplicação referentes aos percentuais de resposta. Outros artigos, que se seguirão a este, irão abordar os resultados relativos aos objetivos descritos.

\section{Materiais e métodos}

\section{Estratégias, população e amostra}

A estratégia geral de delineamento foi a de um estudo epidemiológico seccional (inquérito), com abordagens postal (questionários de auto- preenchimento) e domiciliar (questionários preenchidos por entrevistadores).

A população-alvo era constituída pelos aposentados, com idade de 60 anos ou mais, vinculados ao Regime Geral de Previdência Social do INSS/MPAS. Em 14 de dezembro de 2002, o cadastro da Empresa de Processamento de Dados da Previdência Social (DATAPREV) registrava 10.446.986 brasileiros, com idade igual ou superior a 60 anos, que recebiam benefícios (aposentadoria, pensão ou amparo social ao idoso) previdenciários e assistenciais ativos de prestação continuada pela Previdência Social. Destes, 456.628 eram residentes na Cidade do Rio de Janeiro e 157.809 em Belo Horizonte.

As coletas de informações foram feitas pela abordagem postal em três amostras: no Brasil e nos municípios de Belo Horizonte e do Rio de Janeiro. As informações obtidas diretamente nos domicílios foram feitas em duas amostras: nos municípios de Belo Horizonte e do Rio de Janeiro.

A seleção dos participantes foi realizada pela DATAPREV, com base em seu cadastro de beneficiários, por amostragem aleatória simples, isto é, sem reposição, em novembro de 2002. Para o nível nacional, foram sorteados $3 \mathrm{mil}$ indivíduos para o inquérito de abordagem somente postal. Para os níveis municipais foram sorteados inicialmente 800 indivíduos tanto em Belo Horizonte como no Rio de Janeiro. Nestes municípios estas mesmas amostras selecionadas para os inquéritos postais foram replicadas para os inquéritos domiciliares. Considerando-se um nível de confiança de 95\%, taxas de resposta de $75 \%$ para as entrevistas domiciliares e supondo-se que não existiriam diferenças relevantes entre respondentes e não respondentes, os 600 entrevistados em cada uma das amostras municipais permitiriam obter erros amostrais entre $4 \%$ a $0,8 \%$ para prevalências entre $50 \%$ a $1 \%$, respectivamente. No inquérito postal nacional, mantendo-se a mesma suposição de semelhança entre respondentes e não respondentes, e na expectativa de resposta por parte de um terço dos 3 mil sorteados, os erros amostrais seriam ainda menores para uma amostra efetiva de mil pessoas, de $3,1 \%$ a $0,6 \%$ para prevalências de $50 \%$ a $1 \%$, respectivamente, para um nível de confiança de $95 \%$.

Nos inquéritos domiciliares, para obter quantidades de entrevistas próximas ao planejado foram necessários, ainda, sorteios adicionais, em função da ocorrência de perdas inevitáveis e outras de difícil execução. Foram consideradas perdas inevitáveis aquelas que se referiram aos indivíduos que haviam falecido e 
aos que se mudaram para outros municípios, uma vez que essas pessoas deixaram de fazer parte das populações de referência dos municípios estudados. Outras perdas de difícil execução foram aquelas derivadas dos indivíduos que se mudaram para local desconhecido ou de endereços não localizados. Considerou-se provável que pelo menos a metade destes indivíduos também pudesse ter se mudado para outros municípios ou falecido, especialmente devido ao seu perfil etário. Portanto, às amostras originais foram acrescentadas outras, considerando as somas das perdas inevitáveis com as de difícil execução, e a seleção se deu pelo mesmo processo de amostragem das iniciais. Assim, em Belo Horizonte foi acrescentada uma amostra de 81 indivíduos, e no Rio de Janeiro, de 100 indivíduos. Os indivíduos selecionados nas amostras adicionais não receberam o questionário de autopreenchimento por via postal.

\section{Questionários e variáveis de estudo}

As informações foram obtidas por meio de questionários com perguntas fechadas e précodificadas, exceto aquelas relativas aos medicamentos utilizados. Os questionários de autopreenchimento e para entrevista domiciliar se constituíam em brochuras no tamanho A4. O de autopreenchimento tinha 32 páginas, 21 das quais com as questões, com utilização de cores; o domiciliar tinha 48 páginas, impresso apenas em preto e branco.

Os questionários de autopreenchimento e para entrevista domiciliar contavam com três blocos de perguntas. O primeiro bloco referiase a características sócio-demográficas do participante: data de nascimento, sexo, tipo de moradia, pessoas residentes no domicílio e escolaridade. O segundo bloco referia-se a indicadores das condições de saúde e uso de serviços de saúde: percepção da própria saúde; incapacidade para realizar atividades de rotina por problemas de saúde nas duas últimas semanas; ter estado acamado neste período; número de consultas a médicos nos últimos 12 meses; número de internações hospitalares neste período; queixas quanto à qualidade do atendimento médico; história de doenças diagnosticadas por algum profissional de saúde; utilização de plano de saúde; pagamento e fornecimento de medicamentos por plano de saúde. O terceiro bloco referia-se ao uso de medicamentos: uso regular; independência para o uso de medicamentos no dia-a-dia; gastos com medicamentos no ultimo mês; identificação e caracterização dos medicamentos utilizados nas duas últimas semanas; duração do uso desses medicamentos; origem da prescrição/indicação; local de obtenção; maior problema encontrado quando da necessidade do medicamento; apresentação de comprovante de uso do medicamento; interrupção do uso de algum medicamento que deveria estar sendo usado, apresentando-se o motivo. Ao final de cada bloco, incluiu-se uma variável sobre a necessidade de ajuda ao entrevistado para responder às perguntas do bloco. No questionário para a entrevista domiciliar, além desses blocos, havia dois adicionais, um sobre a qualidade da receita e outro sobre rede e suporte social.

\section{Coleta dos dados}

Os questionários de autopreenchimento foram enviados duas vezes para os endereços dos aposentados sorteados, nos dias 17 de janeiro e 24 de fevereiro de 2003, com porte pago para a devolução da resposta. Para o nível municipal, foram aceitos os questionários preenchidos até a data anterior à abordagem domiciliar, que se iniciou em 19 de março, pouco mais de três semanas após o envio da segunda remessa postal. Para o nível nacional, foram aceitos questionários preenchidos até 31 de maio de 2003.

Os trabalhos de campo dos inquéritos domiciliares se iniciaram em meados de março e se estenderam até junho de 2003 em Belo Horizonte e até agosto de 2003 no Município do Rio de Janeiro.

As entrevistas domiciliares foram feitas, preferencialmente, com os indivíduos selecionados. Entretanto, com aqueles impedidos por motivos de saúde, tais como surdez ou déficit cognitivo, as entrevistas foram realizadas com parentes ou cuidadores, que também prestaram auxílio nos casos de dificuldades com algumas questões, excetuadas aquelas que exigissem auto-avaliação. Nos questionários de autopreenchimento, enviados e retornados pelo correio, também foi solicitado que se assinalasse, em questão específica, sempre que houvesse auxílio de outra pessoa.

Os inquéritos domiciliares foram precedidos por um estudo-piloto para verificar a adequação da dinâmica de recrutamento, testar os instrumentos de coleta de dados e confirmar a viabilidade da investigação.

\section{Equipes de coleta e controle de qualidade dos inquéritos domiciliares}

As coletas domiciliares foram realizadas por entrevistadores graduados ou estudantes, dos últimos períodos, de farmácia. O treinamento 
desses entrevistadores consistiu em provas de simulação e avaliação de desempenho. Para uniformizar o processo de coleta de dados, foi elaborado um Manual de Instruções para Aplicação do Questionário Domiciliar, para consulta. Os entrevistadores tiveram sua produção controlada em períodos semanais, por meio de fichas e planilhas eletrônicas.

O controle de qualidade da tomada de informações foi exercido pela supervisão direta dos dados coletados com a replicação de entrevistas domiciliares em subamostras de até $10 \%$ das entrevistas feitas por cada entrevistador e pelo controle estatístico de algumas informações. Os supervisores de campo foram responsáveis pelas réplicas, pela contínua assistência aos entrevistadores na localização e abordagem prévia dos entrevistados e pela revisão permanente da codificação dos questionários das entrevistas domiciliares. O controle estatístico consistiu na confrontação de alguns dados coletados nessas entrevistas com aqueles constantes nos registros da DATAPREV. Esses sistemas de controle de qualidade visaram a obter dos indivíduos realmente selecionados para participar do estudo as informações corretas, a atingir o maior grau possível de cobertura das amostras, a corrigir erros de conduta, de tomada de informações e a detectar fraudes.

Nos inquéritos domiciliares atuaram 14 entrevistadores em Belo Horizonte e 19 no Rio de Janeiro. Dois supervisores controlaram a coleta em cada uma das localidades.

\section{Organização dos bancos de dados}

Os dados foram organizados em bancos de dados utilizando os programas Paradox ${ }^{\circledR} 4.5$ (Borland International Inc., Scotts Valley, Estados Unidos), Epidata (Epidata Association, Odense, Dinamarca), Epi Info (Centers for Disease Control and Prevention, Atlanta, Estados Unidos) e Stata (Stata Association, College Station, Estados Unidos). Os digitadores foram previamente treinados para incorporação das informações aos bancos de dados, e estes foram organizados separando-se as duas fontes, postal e domiciliar, porém de forma padronizada, de modo a permitir a análise conjunta, quando necessário.

As possibilidades de erros na etapa de processamento de dados foram minimizadas pela validação das entradas de dados em cada variável. Além disso, os bancos foram digitados duas vezes, para permitir confrontação, detecção de erros e posterior correção.

\section{Classificação dos medicamentos}

Para classificar os medicamentos utilizados, os dados foram organizados em tabelas segundo o modelo relacional. Foram utilizadas duas fontes de informação: o DEF (Dicionário de Especialidades Farmacêuticas) 10, com tabelas que relacionam nomes comerciais aos princípios ativos, e a classificação ATC (Anatomical Therapeutic Chemical Classification System) 11, com nomes genéricos e seus códigos.

Uma interface de harmonização foi construída para associar os itens do DEF aos registros originais e, indiretamente, aos princípios ativos das apresentações comerciais. Com esta estratégia, foi possível identificar os registros correspondentes às associações, isto é, com mais de um princípio ativo no mesmo registro.

A identificação dos códigos da ATC para classificação dos princípios ativos foi feita levando-se em consideração três informações: os nomes dos princípios ativos, a dose e a forma farmacêutica. Um campo extra chamado RENAME foi criado para indicar os registros que fazem parte da Relação Nacional de Medicamentos Essenciais. Essa lista foi obtida no sítio da Agência Nacional de Vigilância Sanitária na Internet (http://www.anvisa.gov.br/medicamentos/essencial.htm, acessado em 22/Out/ 2004).

\section{Aspectos éticos}

O projeto foi avaliado e aprovado, inclusive em seus aspectos éticos, pelos Comitês de Ética em Pesquisa da Fundação Oswaldo Cruz e da Universidade Federal de Minas Gerais. Foi garantido o sigilo quanto à identidade dos participantes, assim como a confidencialidade das informações, por meio de carta assinada pela Coordenação Regional do Projeto, que também informava que a participação era voluntária e que a não-participação não acarretaria em nenhum prejuízo na assistência prestada pelo INSS e pelo Sistema Único de Saúde. Consentimentos livres e esclarecidos foram obtidos dos participantes.

\section{Métodos estatísticos}

Neste artigo foram estimadas as estatísticas kappa de Cohen 12, com seus respectivos intervalos de confiança, e estatísticas de $\chi^{2}$ (quiquadrado) para testar independências, inclusive com o uso do método de sua partição ou de seus componentes 13 . Todas as estimativas foram obtidas com o programa Stata. 


\section{Resultados}

A Figura 1 resume as relações entre as populações-alvo, as amostras e os questionários efetivamente preenchidos. Sem considerar os motivos das perdas de questionários, é possível perceber que as abrangências das coberturas pretendidas, tanto nos inquéritos postais como nos domiciliares, foram maiores em Belo Horizonte.

As distribuições dos motivos de perdas de entrevistas nos inquéritos domiciliares podem ser vistas na Tabela 1. Foram consideradas perdas inevitáveis aquelas provocadas por óbitos, mudança de residência para outros municípios e erros de amostragem, estes devidos a sorteio de indivíduos que já residiam fora dos municípios alvos na época da entrevista ou que tivessem menos de 60 anos de idade, apesar do registrado no cadastro da DATAPREV.

Excetuadas as perdas inevitáveis, as coberturas dos inquéritos domiciliares se elevaram para $80,3 \%$ em Belo Horizonte e $70,7 \%$ no Rio de Janeiro. Em Belo Horizonte, o motivo mais comum de perda de entrevista domiciliar foi a não-localização de endereços $(8,9 \%)$, ao passo

Figura 1

Populações, amostras e questionários preenchidos na pesquisa sobre perfil de utilização de medicamentos por aposentados brasileiros, 2003.

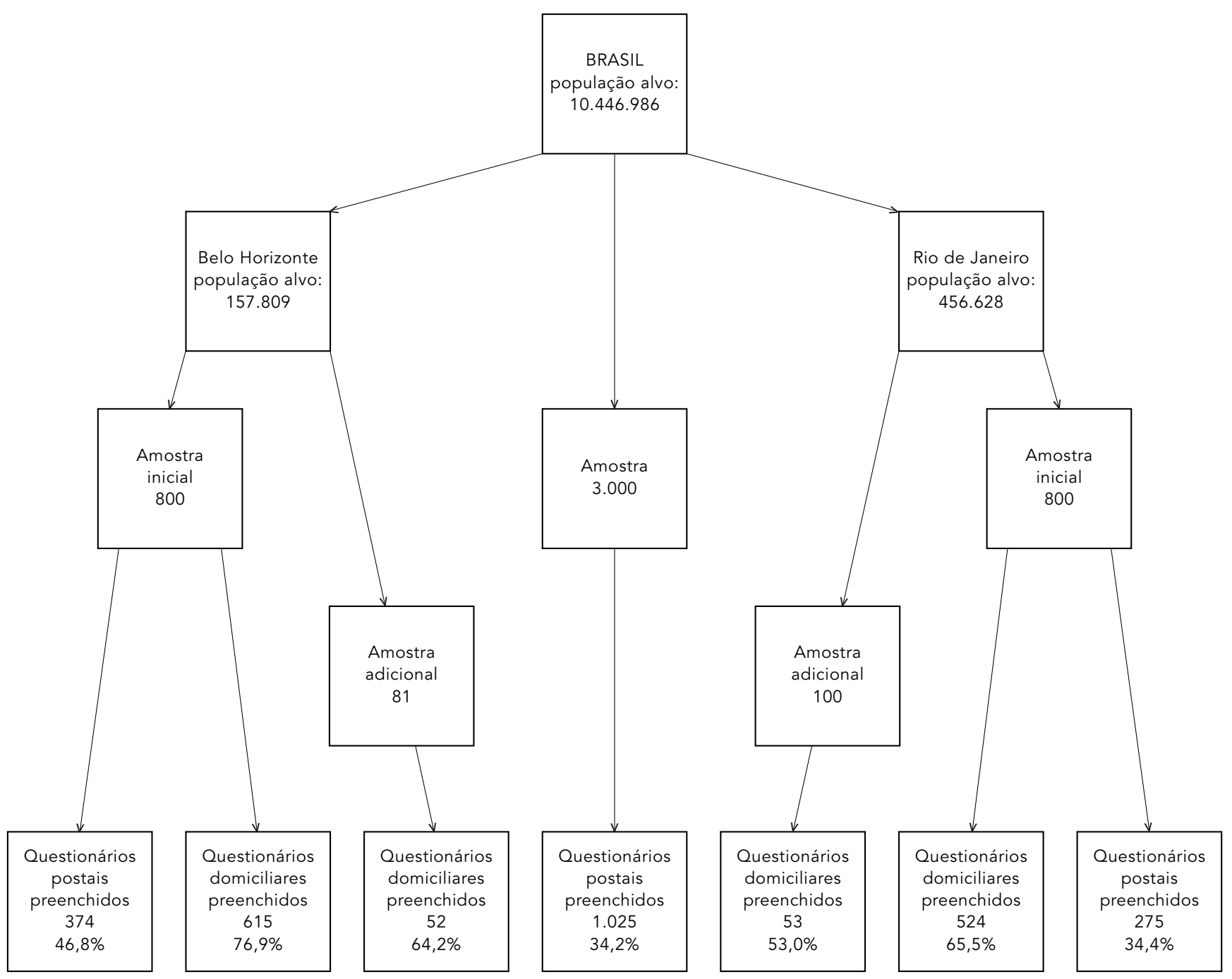


Resultados das entrevistas domiciliares nas amostras dos municípios de Belo Horizonte e do Rio de Janeiro,

Brasil, na pesquisa sobre o perfil de utilização de medicamentos por aposentados, 2003.

\begin{tabular}{|c|c|c|c|c|c|c|c|c|}
\hline \multirow[t]{3}{*}{ Resultados das entrevistas } & \multicolumn{3}{|c|}{ Belo Horizonte } & & \multicolumn{4}{|c|}{ Rio de Janeiro } \\
\hline & \multirow{2}{*}{$\begin{array}{c}\text { Amostra } \\
\text { inicial }\end{array}$} & \multirow{2}{*}{$\begin{array}{l}\text { Amostra } \\
\text { adicional }\end{array}$} & \multicolumn{2}{|c|}{ Total } & \multirow{2}{*}{$\begin{array}{c}\text { Amostra } \\
\text { inicial }\end{array}$} & \multirow{2}{*}{$\begin{array}{l}\text { Amostra } \\
\text { adicional }\end{array}$} & \multicolumn{2}{|c|}{ Total } \\
\hline & & & $n$ & $\%$ & & & $\mathrm{n}$ & $\%$ \\
\hline Realizadas & 615 & 52 & 667 & 80,3 & 524 & 53 & 577 & 70,7 \\
\hline Recusas & 45 & 4 & 49 & 5,9 & 77 & 16 & 93 & 11,4 \\
\hline Não localizados por mudança & 16 & 1 & 17 & 2,0 & 65 & 9 & 74 & 9,1 \\
\hline Endereços não localizados & 62 & 12 & 74 & 8,9 & 34 & 10 & 44 & 5,4 \\
\hline Outros motivos* & 19 & 5 & 24 & 2,9 & 25 & 3 & 28 & 3,4 \\
\hline Perdas evitáveis & 142 & 22 & 164 & 19,7 & 201 & 38 & 239 & 29,3 \\
\hline Amostra efetiva & 757 & 74 & 831 & 100,0 & 725 & 91 & 816 & 100,0 \\
\hline Óbitos & 11 & 1 & 12 & & 22 & 3 & 25 & \\
\hline Mudanças para outros municípios & 28 & 5 & 33 & & 51 & 6 & 57 & \\
\hline Sorteios indevidos & 4 & 1 & 5 & & 2 & 0 & 2 & \\
\hline Perdas inevitáveis & 43 & 7 & 50 & 5,7 & 75 & 9 & 84 & 9,3 \\
\hline Total & 800 & 81 & 881 & 100,0 & 800 & 100 & 900 & 100,0 \\
\hline
\end{tabular}

*Viagem, hospitalização, fora de casa.

que as recusas se constituíram em apenas 5,9\% destas perdas. Já no Rio de Janeiro, as perdas domiciliares mais importantes se deveram às recusas $(11,4 \%)$ e à não-localização de indivíduos por mudança para local desconhecido $(9,1 \%)$.

A Tabela 2 relaciona a obtenção de respostas nos inquéritos domiciliares e postais em Belo Horizonte e no Rio de Janeiro, limitados aos sorteados nas amostras iniciais. Cerca de $5 \%$ dos sorteados só responderam ao inquérito postal em ambas as capitais.

Em Belo Horizonte, entre aqueles que somente responderam ao inquérito postal, $23 \%$ foram identificados como perdas inevitáveis durante o trabalho de campo da coleta domiciliar, enquanto o mesmo ocorreu em $21 \%$ dos que não responderam a nenhum dos inquéritos, diferença não significativa $\left(\chi^{2}=0,00005\right.$; $\mathrm{p}=0,995)$. No Rio de Janeiro, $37 \%$ dos que responderam apenas ao inquérito postal foram identificados como perdas inevitáveis durante o trabalho de campo da coleta domiciliar, enquanto o mesmo só ocorreu com $26 \%$ dos que não responderam a nenhum dos inquéritos; esta diferença também não foi significativa $\left(\chi^{2}=1,63 ; p=0,201\right)$. No Rio de Janeiro, o percentual de recusa à entrevista domiciliar foi superior nos que não responderam a qualquer inquérito, de $29 \%$, quando comparado com o percentual dos que só responderam ao inquérito postal, de $22 \%$, porém essa diferença não foi significativa $\left(\chi^{2}=0,54 ; p=0,464\right)$. Em Belo Horizonte, estes percentuais se aproximaram mais, com $25 \%$ e $23 \%$ de recusas entre os que não responderam a qualquer inquérito e os que responderam apenas ao postal, respectivamente, sendo esta diferença igualmente não significativa $\left(\chi^{2}=0,015 ; \mathrm{p}=0,902\right)$.

A Tabela 3 apresenta os resultados gerais dos três inquéritos postais. A estratégia de enviar uma segunda remessa de questionários revelou-se importante porque acrescentou $28,5 \%$ de respostas no total recebido. As diferenças entre os percentuais de recepção de novos questionários respondidos após a segunda remessa nas três amostras não foram significativas. Entretanto, o percentual geral de respostas ao inquérito postal em Belo Horizonte, de $47 \%$, foi significativamente maior do que nas outras amostras, do Rio de Janeiro e do Brasil, tendo sido, em ambas, de $34 \%$.

A Tabela 4 relaciona, dos indivíduos sorteados, quatro atributos (sexo, idade, região geoeconômica de residência e valor do benefício) que constavam do cadastro fornecido pela DATAPREV de acordo com a participação ou não no estudo postal nacional.

Entre os que responderam ao inquérito postal, na amostra do Brasil, e os que não responderam, não foram detectadas diferenças significativas nas distribuições dos atributos combinados de sexo e grupos etários, de região de residência e de categorias de valor do benefício recebido. 


\section{Discussão}

A opção pelo processo de amostragem aleatória simples pode ser justificada pelo elevado efeito de delineamento observado na estimação de prevalência de uso de medicamentos. Em uma pesquisa feita em Pelotas, Rio Grande do Sul, Brasil 4, este efeito ficou próximo de três com conglomerados domiciliares, ou seja, nossas amostras estimadas inicialmente em 600 indivíduos, efetivamente entrevistados nas duas capitais, equivaleriam a amostras de 1.800 indivíduos de amostras de conglomerados de domicílios.

Outros inquéritos domiciliares realizados no Brasil com propósitos semelhantes obtiveram percentuais de resposta mais elevados. Em Pelotas 4, de uma amostra de 3.372 adultos de 20 anos ou mais, selecionados por amostragem complexa de conglomerados, $94,4 \%$ participaram de um estudo sobre prevalência e uso de medicamentos. Em Bambuí, Minas Gerais, Brasil 6, em um censo dos indivíduos de 60 anos ou mais, $92,2 \%$ responderam a um questionário geral sobre saúde que envolvia o consumo de medicamentos. Alguns estudos, também limitados a populações de municípios brasileiros com menos de 500 mil habitantes, não referem perdas, de forma explícita 5,8,9, enquanto outro, circunscrito apenas às mulheres de 60 anos inscritas em um serviço universitário de atenção à saúde, refere uma cobertura de 60,9\% 7 . Por outro lado, na Espanha, um inquérito sobre consumo de medicamentos, aplicado em duas unidades móveis, obteve uma taxa de cobertura de $72 \%$ em uma amostra de adultos de 25 a 64 anos de idade 14 , ao passo que, em um inquérito sobre morbidade e uso de serviços de saúde na Holanda, a cobertura domiciliar foi de apenas 56,9\% nos indivíduos de 65 anos ou mais 15 .

No presente estudo, os indivíduos que participaram dos inquéritos domiciliares nas amos- tras iniciais haviam recebido previamente os questionários de autopreenchimento, além da carta de apresentação da pesquisa. Como aqueles das amostras adicionais não receberam o mesmo material previamente ao estudo domiciliar, é possível que seus percentuais de resposta tenham sido prejudicados por essa diferença de tratamento.

É preciso levar em consideração que as seleções amostrais haviam sido realizadas cerca de dois meses antes do início dos inquéritos postais e quatro meses antes dos inquéritos domiciliares. Além disso, o cadastro da DATAPREV, de onde foram retiradas as amostras, possivelmente não sofreu atualizações regulares e homogêneas de acordo com os locais de residên-

Tabela 2

Respostas aos questionários domiciliares e postais na pesquisa sobre o perfil de utilização de medicamentos por aposentados nos municípios de Belo Horizonte e do Rio de Janeiro, Brasil, 2003.

\begin{tabular}{|c|c|c|c|c|c|c|}
\hline & \multicolumn{6}{|c|}{ Postal } \\
\hline & \multicolumn{2}{|c|}{ Respondeu } & \multicolumn{2}{|c|}{ Não respondeu } & \multicolumn{2}{|c|}{ Total } \\
\hline & $\mathrm{n}$ & $\%$ & $\mathrm{n}$ & $\%$ & $\mathrm{n}$ & $\%$ \\
\hline \multicolumn{7}{|l|}{ Belo Horizonte } \\
\hline \multicolumn{7}{|l|}{ Domiciliar } \\
\hline Respondeu & 335 & 41,5 & 280 & 35,0 & 615 & 76,9 \\
\hline Não respondeu & 39 & 4,9 & 146 & 18,3 & 185 & 23,1 \\
\hline Total & 374 & 46,8 & 426 & 53,3 & 800 & 100,0 \\
\hline \multicolumn{7}{|l|}{ Rio de Janeiro } \\
\hline \multicolumn{7}{|l|}{ Domiciliar } \\
\hline Respondeu & 234 & 29,3 & 290 & 36,3 & 524 & 65,5 \\
\hline Não respondeu & 41 & 5,1 & 235 & 29,4 & 276 & 34,5 \\
\hline Total & 275 & 34,4 & 525 & 65,6 & 800 & 100,0 \\
\hline
\end{tabular}

Belo Horizonte: kappa de Cohen = 0,23 (IC95\%: 0,17-0,29);

Rio de Janeiro: kappa de Cohen =0,25 (IC95\%: 0,19-0,30).

Tabela 3

Respostas aos questionários postais enviados na pesquisa sobre o perfil de utilização de medicamentos por aposentados nas amostras dos municípios de Belo Horizonte e do Rio de Janeiro e do Brasil, 2003.

\begin{tabular}{llccrr}
\hline Resposta & Remessa & Belo Horizonte & Rio de Janeiro & Brasil & Total \\
\hline Respondeu & 1ạ remessa & 268 & 211 & 718 & 1.197 \\
& Só 2ạ remessa & 106 & 64 & 307 & 477 \\
Não respondeu & & 426 & 525 & 1.975 & 2.926 \\
Total & & 800 & 800 & 3.000 & 4.600 \\
\hline
\end{tabular}

Total: $\chi^{2}$ (4g.l.) $=49,39 ; p<0,001$;

1a remessa X só 2a remessa: $\chi^{2}(2 \mathrm{~g} . \mathrm{l})=4,.75 ; \mathrm{p}=0,093$.

Respondeu X não respondeu: $\chi^{2}$ (2g.l.) $=44,90 ; p<0,001$ 
Tabela 4

Sexo, grupos etários, região geo-econômica de residência e valor do benefício dos que participaram ou não na pesquisa sobre o perfil de utilização de medicamentos por aposentados brasileiros na amostra postal do Brasil, 2003.

\begin{tabular}{|c|c|c|c|c|c|}
\hline \multirow[t]{2}{*}{ Variável* } & \multicolumn{2}{|c|}{ Respondeu } & \multicolumn{2}{|c|}{ Não respondeu } & \multirow{2}{*}{$\begin{array}{c}\text { Total } \\
\mathrm{n}\end{array}$} \\
\hline & $\mathrm{n}$ & $\%$ & $\mathrm{n}$ & $\%$ & \\
\hline \multicolumn{6}{|l|}{ Sexo/idade (anos) } \\
\hline Masculino/60-69 & 225 & 22,0 & 419 & 21,3 & 644 \\
\hline Masculino/70 ou + & 193 & 18,9 & 411 & 20,9 & 604 \\
\hline Feminino/60-69 & 289 & 28,3 & 549 & 27,9 & 838 \\
\hline Feminino/70 ou + & 314 & 30,8 & 591 & 30,0 & 905 \\
\hline \multicolumn{6}{|l|}{ Região } \\
\hline Norte & 33 & 3,2 & 87 & 4,4 & 120 \\
\hline Nordeste & 273 & 26,6 & 521 & 26,4 & 794 \\
\hline Centro-Oeste & 38 & 3,7 & 78 & 4,0 & 116 \\
\hline Sudeste & 487 & 47,5 & 941 & 47,7 & 1.428 \\
\hline Sul & 194 & 18,9 & 347 & 17,6 & 541 \\
\hline \multicolumn{6}{|l|}{ Valor do benefício } \\
\hline$\leq \mathrm{R} \$ 200,00 * \star$ & 696 & 67,9 & 1.392 & 70,5 & 2.088 \\
\hline$>\mathrm{R} \$ 200,00 * *$ & 329 & 32,1 & 583 & 29,5 & 912 \\
\hline
\end{tabular}

* Informações do cadastro do Instituto Nacional de Seguridade Social/

Empresa de Processamento de Dados da Previdência Social INSS/DATAPREV;

** Mediana do benefício na época da pesquisa: $\mathrm{R} \$ 200,00$

Sexo-idade: $\chi^{2}$ (3g.I.) $=1,64 ; p=0,651$;

Região geo-econômica: $\chi^{2}$ (4g.l.) $=3,18 ; p=0,528$;

Valor do benefício: $\chi^{2}$ (1 g.I.) $=2,12 ; \mathrm{p}=0,14$.

cia dos beneficiários do INSS. O registro dos óbitos, assim como as declarações de mudança para outros municípios, foram feitas de acordo com as informações prestadas por familiares ou vizinhos, não tendo sido feita verificação de óbitos no sistema oficial de registro. Porém, é possível, ainda, que uma parte dos não localizados, sem qualquer referência, tenha se mudado para outros municípios ou até mesmo falecido. De qualquer modo, os cálculos das taxas de cobertura nos inquéritos domiciliares devem excluir as perdas registradas por motivos inevitáveis.

Não é possível fazer as mesmas considerações, com segurança, para os inquéritos postais, porque os únicos indícios de retorno sem resposta foram os fornecidos pela empresa dos Correios, cujos critérios são diferentes dos adotados nesta pesquisa. Assim, para os Correios, erros no código de endereçamento postal e destinatário ausente são motivos de devolução, contudo algumas vezes, com freqüência relativa desconhecida, a correspondência é entregue a terceiros sem verificações adicionais. De qualquer forma, após a primeira remessa dos questionários de autopreenchimento, foram devolvidos pelos Correios 4,9\%, 6,8\% e 20,3\% dos envelopes enviados para Belo Horizonte, Rio de Janeiro e Brasil, respectivamente. Repare-se que, no caso da amostra postal nacional, as perdas iniciais, inevitáveis, de pelo menos um quinto dos questionários enviados fariam com que a cobertura real pudesse ser estimada em mais de $40 \%$.

Os índices de concordância na obtenção de respostas pelos dois métodos de abordagem, postal e domiciliar, foram semelhantes nas duas capitais, porém baixos. A baixa concordância se deveu principalmente às elevadas freqüências daqueles que responderam ao inquérito domiciliar, mas não ao postal.

As perdas de entrevistas domiciliares por recusa não parecem estar relacionadas com o fato de esses inquéritos terem sido precedidos pelos postais, em ambas as capitais, uma vez que o percentual de recusas domiciliares não diferiu significativamente entre os que foram entrevistados apenas no inquérito postal e aqueles que foram perdidos em ambos os inquéritos, em Belo Horizonte e no Rio de Janeiro.

Os resultados apresentados na Tabela 2 poderiam suportar a suposição de que o inquérito domiciliar no Rio de Janeiro teria sido menos eficiente na cobertura do que o realizado em Belo Horizonte. Por outro lado, a cobertura domiciliar, comparada à postal, foi relativamente maior no Rio de Janeiro $(1,91 x)$ do que em Belo Horizonte $(1,64 x)$, o que poderia indicar maior eficiência relativa no inquérito domiciliar daquela cidade. Se a cobertura do inquérito postal é um dos indicativos da probabilidade de encontro das unidades amostrais em seus endereços, combinado à propensão à resposta a qualquer abordagem, é possível justificar por que seria esperado um maior índice de cobertura no inquérito domiciliar em Belo Horizonte. Uma análise mais extensa das relações entre os inquéritos domiciliares e postais, com avaliações de confiabilidade, será apresentada em artigo específico.

Os percentuais de resposta aos inquéritos postais, em Belo Horizonte, no Rio de Janeiro e no Brasil, podem ser considerados relativamente baixos quando comparados aos encontrados em estudos que aplicaram esta abordagem em outros países. Todavia, os índices de cobertura de inquéritos postais sobre saúde realizados em outros países apresentaram grandes variações. Em uma amostra na Noruega, por exemplo, a cobertura de resposta a um questionário de autopreenchimento, devolvido pelo correio, foi de $91,7 \%$ entre mulheres de 20 a 61 anos que haviam sido atendidas previamente para realização de exames complementares 16. Já em um estudo postal, nos Estados Unidos, apenas $41 \%$ dos mais velhos, de 65 anos ou mais, responderam 17 . 
Em um artigo de revisão sobre as taxas de resposta a inquéritos postais publicados em periódicos médicos dos Estados Unidos, em 1991, a média ficou em $60 \%$, variando de acordo com o assunto e as técnicas utilizadas 18. Na Europa, um estudo sobre prescrição de medicamentos e seus determinantes em mulheres de 35 a 65 anos obteve retorno postal de $71,2 \%$, na Suécia 19 , e outro sobre prevalência de medicamentos não apropriados em pessoas com 75 anos ou mais, na Finlândia, conseguiu 78\% de respostas pela via postal 20 .

Em alguns estudos houve a preocupação de investigar os possíveis determinantes das taxas de resposta aos inquéritos postais. Em estudo com consumidores de plano de saúde nos Estados Unidos, os mais velhos, os incapacitados e os de menor grau de educação tinham taxas de resposta discretamente menores 21 . Já em outro estudo, no Canadá, não foram encontradas diferenças entre respondentes e não respondentes em relação a status sócio-econômico, distribuição étnica, idade, sexo, educação 22 . O estudo na Holanda corroborou essas observações, acrescentando semelhanças no estado conjugal, região de residência, composição familiar, trabalho e índice de massa corporal, mas encontrou discreta diferença no grau de educação, sendo que os menos educados pareciam menos representados no estudo postal 15 .

O tamanho do questionário de autopreenchimento enviado no presente estudo pode ser considerado longo e, portanto, motivo para baixa cobertura de resposta. Ainda assim, outro estudo, feito nos Estados Unidos, que enviou questionários de tamanhos diferentes (de 4 páginas e 16 itens, e de 16 páginas e 76 itens), não encontrou diferenças nas taxas de respostas enviadas pelo correio, inclusive dos mais velhos, de 65 anos ou mais 17. Neste mesmo estudo, também ficou evidente que uma segunda remessa do questionário postal foi melhor do que enviar um cartão postal de lembrança. Por sinal, esta resposta à segunda remessa do questionário implicou aumento de $27,4 \%$ da taxa de resposta, índice muito próximo ao alcançado no presente estudo com a mesma medida (28,5\% no geral) (Tabela 3$)$.

Uma baixa cobertura de respostas não significa necessariamente baixa representatividade. Embora existam maiores oportunidades de viés de não-resposta em inquéritos com baixas taxas de resposta, estes podem prover amostras cuja representatividade da população de interesse é até maior do que aquelas obtidas em inquéritos com altas taxas de resposta 18 . O óbice mais importante em um inquérito de baixas taxas de resposta pode estar relacionado apenas com as perdas de precisão das estimativas desejadas. No presente estudo, as amostras planejadas foram superdimensionadas a fim de se obterem amostras efetivamente observadas que permitissem atingir as precisões desejadas, supondo-se que os respondentes representam as populações de referência.

$\mathrm{O}$ achado das semelhanças de alguns atributos entre os que responderam ao inquérito postal, na amostra do Brasil, e os que não responderam, se não permite concluir que a amostra dos que responderam pode ser de fato representativa do total, também não permite afastar essa hipótese. Outros atributos, investigados nos questionários deste estudo e nos de outros já citados, como grau de instrução, não estão disponíveis nas informações cedidas pela DATAPREV para esta pesquisa, de modo que a comparação entre respondentes e não respondentes teve de se limitar ao apresentado na Tabela 4. Ainda assim, na ausência de outros estudos de abrangência nacional sobre o tema investigado, iremos utilizar esta amostra para inferir resultados para a população-alvo, os aposentados brasileiros de 60 anos ou mais, beneficiários do sistema público (INSS).

\section{Resumo}

São relatados aspectos metodológicos e coberturas de resposta em estudo cujo objetivo principal era descrever o perfil de utilização de medicamentos por aposentadose pensionistas do Instituto Nacional de Seguridade Social, de 60 anos ou mais, em três amostras aleatórias simples: do Brasil e dos municípios de Belo Horizonte e do Rio de Janeiro. Trata-se de estudo epidemiológico seccional, com abordagens postal (questionários de autopreenchimento) e domiciliar (questionários preenchidos por entrevistadores). Esta estratégia permitirá avaliar a concordância de respostas obtidas em ambas as abordagens. $\mathrm{Na}$ amostra nacional de 3.000 indivíduos, 1.025 responderam apenas ao questionário de autopreenchimento. Ape- sar das perdas consideráveis, os respondentes, pelas semelhanças com os não respondentes, parecem constituir amostra representativa da população-alvo. Em Belo Horizonte e no Rio de Janeiro, foram replicadas as amostras iniciais de 800 indivíduos para as abordagens postal e domiciliar. As coberturas de respostas postais foram de 46,8\% e de 34,4\% em Belo Horizonte e no Rio de Janeiro, respectivamente. Nos inquéritos domiciliares, os percentuais de resposta foram de 80,3\% e de 70,7\% em Belo Horizonte e no Rio de Janeiro, respectivamente, expurgadas as perdas por motivos inevitáveis.

Entrevistas; Saúde do Idoso; Uso de Medicamentos 


\section{Colaboradores}

F. A. Acurcio e S. Rozenfeld, coordenadores da pesquisa, participaram de seu planejamento, supervisão de coleta e análise dos dados. A. Q. Ribeiro e C. R. Andrade participaram do planejamento, da supervisão de coleta e da análise dos dados. C. H. Klein colaborou no planejamento, supervisão de coleta, análise dos dados e redação e revisão do manuscrito deste artigo. C. S. Moura contribuiu no planejamento, supervisão de coleta e prestou assessoria técnica na constituição dos bancos de dados.

\section{Referências}

1. Gordilho A, Sérgio J, Silvestre J, Ramos LR, Freire MPA, Espindola N, et al. Desafios a serem enfrentados no terceiro milênio pelo setor saúde na atenção integral ao idoso. Rio de Janeiro: Universidade Aberta da Terceira Idade, Universidade do Estado do Rio de Janeiro; 2000.

2. Ministério da Previdência e Assistência Social. Gestão quadripartite da Previdência Social. Relatório gerencial. Brasília: Ministério da Previdência e Assistência Social; 2002.

3. Rozenfeld S. Prevalência, fatores associados e mau uso de medicamentos entre os idosos: uma revisão. Cad Saúde Pública 2003; 19:717-24.

4. Bertoldi AD, Barros AJD, Hallal PC, Lima RC. Utilização de medicamentos em adultos: prevalência e determinantes individuais. Rev Saúde Pública $2004 ; 38: 228-38$.

5. Loyola Filho AI, Uchoa E, Guerra HL, Firmo JOA, Lima-Costa MF. Prevalência e fatores associados à automedicação: resultados do projeto Bambuí. Rev Saúde Pública 2002; 36:55-62.

6. Lima-Costa MF, Uchoa E, Guerra HL, Firmo JOA, Vidigal PG, Barreto SM. The Bambuí health and ageing study (BHAS): methodological approach and preliminary results of a population-based cohort study of the elderly in Brazil. Rev Saúde Pública 2000; 34:126-35.

7. Rozenfeld S, Camacho LAB, Veras RP. Medication as a risk factor for falls in older women in Brazil. Rev Panam Salud Pública 2003; 13:369-75.

8. Vilarino JF, Soares IC, Silveira CM, Rödel APP, Bortoli R, Lemos RR. Perfil da automedicação em município do Sul do Brasil. Rev Saúde Pública 1998; 32:43-9.

9. Simões MJS, Farache Filho A. Consumo de medicamentos em região do Estado de São Paulo (Brasil), 1985. Rev Saúde Pública 1988; 22:494-9.

10. Dicionário de Especialidades Farmacêuticas. 29a Ed. Rio de Janeiro: Editora de Publicações Científicas; 2000.

11. WHO Collaborating Centre for Drug Statistics Methodology. Anatomical Therapeutic Chemical (ATC) classification index with Defined Daily Doses (DDD's). Oslo: WHO Collaborating Centre for Drug Statistics Methodology; 2000.

\section{Agradecimentos}

Este estudo foi financiado pelo Fundo Nacional de Saúde, Ministério da Saúde, através da então Gerência Técnica de Assistência Farmacêutica/Departamento de Atenção Básica de Saúde/Secretaria de Políticas de Saúde (GTAF/DAB/SPS - convênio n. 796/ 2002). Também recebeu apoio logístico da Faculdade de Farmácia, Universidade Federal de Minas Gerais; da Escola Nacional de Saúde Pública Sergio Arouca e da Casa de Oswaldo Cruz, ambos da Fundação Oswaldo Cruz. Os autores agradecem a colaboração de Maria Fernanda Furtado de Lima-Costa, Henrique Leonardo Guerra, Carlos Alberto Pereira Gomes, Luiz Antônio Marinho e Geraldo Adão Santos.

12. Fleiss JL, Levin B, Paik MC. Statistical methods for rates and proportions. Hoboken: John Wiley \& Sons; 2003.

13. Armitage P, Berry G. Statistical methods in medical research. Oxford: Blackwell; 1987.

14. Sans S, Paluzie G, Puig T, Balaña L, Balaguer-Vintró I. Prevalencia del consumo de medicamentos em la población adulta de Catalunya. Gac Sanit 2002; 16:121-30.

15. Picavet HSJ. National health surveys by mail or home interview: effects on response. J Epidemiol Community Health 2001; 55:408-13.

16. Eggen AE. Pattern of drug use in a general population - prevalence and predicting factors: the Tromso study. Int J Epidemiol 1994; 23:1262-72.

17. Hoffman SC, Burke AE, Helzlsouer KJ, Comstock GW. Controlled trial of the effect of length, incentives, and follow-up techniques on response to a mailed questionnaire. Am J Epidemiol 1998; 148: 1007-11.

18. Asch DA, Jedrziewski MK, Christakis NA. Response rates do mail surveys published in medical journals. J Clin Epidemiol 1997; 50:1129-36.

19. Bardel A, Wallander MA, Svärdsudd K. Reported current use of prescription drugs and som of its determinants among 35 to 65 -year-old women in Sweden: a population-based study. J Clin Epidemiol 2000; 53:637-43.

20. Pitkala KH, Strandberg TE, Tilvis RS. Inappropriate drug prescription in home-dwelling, elderly patients: a population-based survey. Arch Intern Med 2002; 162:1707-12.

21. Zaslavsky AM, Zaborski LB, Cleary PD. Factors affecting response rates to the consumer assessment of health plans study survey. Med Care 2002; 40:485-99.

22. Siemiatycky J, Campbell S. Nonresponse bias and early versus all responders in mail and telephone surveys. Am J Epidemiol 1984; 120:291-301.

Recebido em 12/Nov/2004

Versão final reapresentada em 06/Jun/2005

Aprovado em 13/Jun/2005 